\title{
Virtual geometricity is rare
}

\author{
Christopher H. Cashen and Jason F. Manning
}

\begin{abstract}
We present the results of computer experiments suggesting that the probability that a random multiword in a free group is virtually geometric decays to zero exponentially quickly in the length of the multiword. We also prove this fact.
\end{abstract}

\section{Introduction}

Let $\mathbb{F}$ be a finite rank non-abelian free group. Fix, once and for all, a basis $\underline{x}=\left\{x_{1}, \ldots, x_{r}\right\}$. A word in $\mathbb{F}$ is an element of $\mathbb{F}$ expressed as a freely reduced word in the letters $\underline{x}^{ \pm}$. A multiword $\underline{w}=\left\{w_{1}, \ldots, w_{k}\right\}$ is a finite subset of $\mathbb{F}$. The set $\underline{w}$ determines a collection of conjugacy classes $[\underline{w}]=\left\{\left[w_{1}\right], \ldots,\left[w_{k}\right]\right\}$ in $\mathbb{F}$, possibly with multiplicity.

DEFinition 1.1. Fix an identification of $\mathbb{F}$ with the fundamental group of an orientable 3 -dimensional handlebody $H$. The set of conjugacy classes $[\underline{w}]$ determines a free homotopy class of map $\bigsqcup_{w \in w} S^{1} \rightarrow H$. The multiword $\underline{w}$ is (orientably) geometric if the homotopy class determined by $[\underline{w}]$ contains an embedding into $\partial H$.

Similarly, a multiword $\underline{w}$ is non-orientably geometric if there is such an embedding where we allow $H$ to be a non-orientable handlebody.

REMark. Geometricity does not depend on the choice of identification $\mathbb{F}=\pi_{1}(H)$, since the handlebody group, that is, the group of (orientation preserving) homeomorphisms of $H$ modulo isotopy, surjects onto the outer automorphism group of $\pi_{1}(H)$.

Definition 1.2. If $[w]$ is a conjugacy class in $\mathbb{F}$ and $F<\mathbb{F}$ is a finite index subgroup, we can 'lift' $[w]$ to $F$ as follows. Let $[w]_{F}$ be the set of $F$-conjugacy classes of the form $g^{-1} w^{\alpha} g$, where $g \in \mathbb{F}$ and $\alpha=\alpha(w, g) \geqslant 1$ is minimal subject to the requirement that $g^{-1} w^{\alpha} g \in F$. The lift $[\underline{w}]_{F}$ of $[\underline{w}]$ to $F$ is then defined to be $\bigsqcup_{w \in \underline{w}}[w]_{F}$.

(From a topological point of view, let $\tilde{H} \rightarrow H$ be the cover corresponding to $F<\mathbb{F}$. A conjugacy class $[w]$ corresponds to the free homotopy class of some map $\phi: S^{1} \rightarrow H$, and $[w]_{F}$ corresponds to the collection of free homotopy classes of elevations of $\phi$ to $\tilde{H}$.)

Definition 1.3. A multiword $\underline{w}$ is virtually geometric if there exists a finite index subgroup $F$ of $\mathbb{F}$ such that $[\underline{w}]_{F}$ is geometric.

Received 11 September 2014; revised 16 March 2015.

2010 Mathematics Subject Classification 20E05, 20P05, 57M10 (primary).

The first author was supported by the Agence Nationale de la Recherche (ANR) grant ANR-2010-BLAN116-01 GGAA, the European Research Council (ERC) grant of Goulnara ARZHANTSEVA, grant agreement \#259527, and the ICERM Program 'Low-dimensional Topology, Geometry, and Dynamics'. The second author was partly supported by the National Science Foundation, grants DMS 1104703 and 1343229. This project also benefited greatly from a visit funded by the GEAR network, grants DMS 1107452, 1107263, 1107367. 
The Baumslag-Solitar words $\bar{b} a^{p} b \bar{a}^{q}$ in $\mathbb{F}_{2}=\langle a, b\rangle$ with $p \neq 0 \neq q$ and $|p| \neq|q|$ are examples of words that are virtually geometric but not geometric $[\mathbf{7}, \S 6]$.

If $\underline{w}$ is orientably geometric then $\langle\underline{x} \mid \underline{w}\rangle$ is the fundamental group of a compact 3-manifold. This can be seen by taking the handlebody $H$ with $\pi_{1}(H)=\mathbb{F}$ and attaching a 2-handle along each component of a regular neighborhood of the embedded multicurve representing $[\underline{w}]$.

If $\underline{w}$ is virtually geometric then there exists a positive number $n$ such that $\langle\underline{x}| w^{n}$ for $\left.w \in \underline{w}\right\rangle$ is virtually a 3-manifold group (see [15, Remark 1.5]).

Gordon and Wilton [7] studied virtual geometricity as an approach to a special case of Gromov's surface subgroup conjecture. They show that if $\underline{w}$ is virtually geometric, then the double of $\mathbb{F}$ along $\underline{w}$, constructed by taking two copies of $\mathbb{F}$ and identifying the two copies of each $w \in \underline{w}$ via cyclic amalgamation, is virtually a 3 -manifold group, and contains a surface subgroup when it is 1-ended. In an early version of [7] ([6, Question 22]), they ask whether any non-virtually geometric word exists. One reason to suspect words to be always virtually geometric is the effectiveness in low-dimensional topology of arguments 'desingularizing' immersed submanifolds in finite-sheeted covers. Two important examples of such arguments are Papakyriakopoulos' 'Tower argument' for the loop and sphere theorems [17] and Scott's argument for subgroup separability in surface groups [19]. However, there do exist non-virtually geometric words; the second author gave a (non-generic) criterion in [15] and exhibited some words which satisfy it.

One might still wonder how common virtual geometricity is. We wrote a computer program that determines if a given multiword is virtually geometric or not, and set it to work testing random multiwords in low rank free groups. Our experiments, presented in $\S 7$, suggest that the probability that a random multiword is virtually geometric decays to zero exponentially quickly in the length of the multiword. We also make explicit estimates for the rate of exponential decay. Surprisingly, our experiments suggest that the ratio of the number of virtually geometric words to the number of geometric words of a given length is bounded above.

QUESTION. Does the ratio of virtually geometric words to geometric words stay bounded as the length goes to infinity? Does it tend to 1 ?

In $\S \S 3-6$ of the paper we apply the technology developed in $[\mathbf{3}, 4]$ to establish the result suggested by the experiments. In the terminology of $\S 2$, we show the following theorem.

\section{TheOREM. Virtual geometricity is exponentially rare.}

More precisely, recall that $\mathbb{F}$ is a free group of fixed finite rank $r>1$. In Theorem 6.3 we show that the proportion of words of length $l$ in $\mathbb{F}$ which are virtually geometric decays to zero exponentially quickly in $l$. The same is true if we restrict our attention to the subset $\mathbb{C} \subseteq \mathbb{F}$ consisting of cyclically reduced words.

For multiwords there are different models of genericity. However, a multiword that contains a non-virtually geometric word is itself non-virtually geometric, so virtual geometricity will also be exponentially rare for any model in which a random multiword contains a long random word. In particular, virtual geometricity is exponentially rare for multiwords in both the 'few relators model' of genericity (Corollary 6.4) and the 'density model' (Corollary 6.5).

The rough idea of the proof is to find a 'poison' word $v \in \mathbb{F}$ which obstructs the virtual geometricity of any cyclically reduced $w \in \mathbb{F}$ containing $v$ as a subword.

The first author $[\mathbf{3}]$ characterized virtually geometric multiwords as those that are constructed as amalgams of geometric pieces. In particular, if $\mathbb{F}$ does not admit cyclic splittings relative to $\underline{w}$, that is, splittings in which the elements of $\underline{w}$ are elliptic, then virtual geometricity reduces to geometricity. The poison word $v$ is a concatenation of words $v_{1}$ and $v_{2}$ so that $v_{1}$ obstructs the existence of a relative splitting, and $v_{2}$ obstructs non-orientable geometricity. The characterization from [3] implies that $v=v_{1} v_{2}$ obstructs virtual geometricity. 
Finally we appeal to the well-known fact (Proposition 2.3) that cyclically reduced words exponentially generically contain every short word - in particular they contain $v$.

In fact there is the slight complication that our word $v$ is only poisonous to Whitehead minimal words, but these are exponentially generic by a result of Kapovich-Schupp-Shpilrain $[12]$.

\section{Generic sets}

Our definitions in this section follow Kapovich, Schupp, and Shpilrain [12]. A sequence $\left(c_{n}\right) \subset$ $\mathbb{R}$ with $\lim _{n \rightarrow \infty} c_{n}=c \in \mathbb{R}$ converges exponentially fast if there exist $a>0$ and $b \in \mathbb{R}$ such that $\left|c-c_{n}\right| \leqslant \exp (b-a n)$ for all sufficiently large $n$.

Let $|w|$ denote the length of $w$ in the word metric on $\mathbb{F}$ corresponding to $\underline{x}$.

Definition 2.1. Let $A \subset B \subset \mathbb{F}$. The set $A$ is generic in $B$ if

$$
\lim _{n \rightarrow \infty} \frac{\#\{w \in A|| w \mid \leqslant n\}}{\#\{w \in B|| w \mid \leqslant n\}}=1 .
$$

$A$ is exponentially generic in $B$ if the convergence is exponentially fast.

A subset is rare, or negligible, if the complement is generic. It is exponentially rare, or exponentially negligible, if the complement is exponentially generic.

It is an easy computation to see that the intersection of finitely many (exponentially) generic sets is (exponentially) generic.

A property $\mathcal{P}$ is said to be (exponentially) generic/rare in $B$ if the set of words having $\mathcal{P}$ is (exponentially) generic/rare.

Let $\mathbb{C}$ be the set of cyclically reduced words in $\mathbb{F}$ with respect to the basis $\underline{x}$.

TheOREm 2.2 [12, Theorem B(1)]. The set of cyclically reduced words that are not proper powers and are Whitehead minimal is exponentially generic in $\mathbb{C}$. The set of words that are not proper powers and whose cyclic reduction is Whitehead minimal is exponentially generic in $\mathbb{F}$.

Proposition 2.3. Let $w$ be a word in $\mathbb{F}$. The subset of words that contain $w$ as a subword is exponentially generic in $\mathbb{F}$. The subset of cyclically reduced words that contain $w$ as a subword is exponentially generic in $\mathbb{C}$.

Proof. This fact is well known. For $\mathbb{F}$, see $[\mathbf{8}, \S 2]$ or $[\mathbf{1 3}$, Corollary 4.4.9]. For $\mathbb{C}$, see $[\mathbf{1 4}$, Lemma 2.5]. Note that the statement of the latter result includes the assumption that $|w|>4$, but in fact the estimate and proof given there are valid for any subword $w$ once the random word length is greater than 16 .

It is also true that for a fixed word $w \in \mathbb{F}$ the set of words that contain $w$ as a subword of their cyclic reduction is exponentially generic. This can be deduced from [12, Proposition 6.2], which is stated without proof. For completeness, we give a proof in Proposition 2.8. The proof uses a few auxiliary lemmas.

Lemma 2.4. Let $A \subset \mathbb{F}$. Let $S_{n}$ be the set of words in $\mathbb{F}$ of length exactly $n$, and suppose there exist $a>0, b \in \mathbb{R}$ so that

$$
\frac{\# A \cap S_{n}}{\# S_{n}} \leqslant \exp (b-a n)
$$

for all sufficiently large $n$. Then $A$ is exponentially rare in $\mathbb{F}$. 
Proof. In fact one can show that for any $0<a^{\prime}<\min \{a, \ln (2 r-1)\}$, there is a $b^{\prime}$ so that $\left(\# A \cap B_{n}\right) / \# B_{n} \leqslant \exp \left(b^{\prime}-a^{\prime} n\right)$. This straightforward computation is left to the reader.

Let $\lceil x\rceil$ denote the least integer greater than or equal to $x$.

Definition 2.5. Define the middle third of a word $w \in \mathbb{F}$ of length $l \geqslant 5$ to be the subword of $w$ obtained by discarding the first and last $\lceil l / 3\rceil$ letters.

Lemma 2.6. Let $w$ be a word in $\mathbb{F}$. The set of words containing $w$ as a subword of their middle third is exponentially generic in $\mathbb{F}$.

Proof. The ratio of the number of words of length $l$ not containing $w$ as a subword of their middle third to the number of words of length $l$ is equal to the ratio of the number of words of length $m=l-2\lceil l / 3\rceil$ not containing $w$ as a subword to the number of words of length $m$. By Proposition 2.3, this ratio decays exponentially in $m$, but $m$ is linear in $l$, so the ratio decays exponentially in $l$. Conclude by applying Lemma 2.4 .

Lemma 2.7. The set of words $w \in \mathbb{F}$ such that the middle third of $w$ is a subword of the cyclic reduction of $w$ is exponentially generic in $\mathbb{F}$.

Proof. Let $r$ be the rank of $\mathbb{F}$. Let $n(l)$ be the number of words of length $l$ for which cyclic reduction reduces length by at least $2\lceil l / 3\rceil$. Every such word is of the form $w v w^{-1}$, where $w$ is of length $\lceil l / 3\rceil, v$ is of length $m=l-2\lceil l / 3\rceil$, and the last letter of $w$ is not the inverse of the first letter of $v$. Thus, $n(l) \leqslant 2 r(2 r-1)^{(m-1)} \cdot(2 r-1)^{\lceil l / 3\rceil}$. In fact, the inequality is strict because for some choices there will be a free reduction in $v w^{-1}$, resulting in a word of length less than $l$. We have

$$
\frac{n(l)}{2 r(2 r-1)^{(l-1)}}<\frac{2 r(2 r-1)^{(m-1)} \cdot(2 r-1)^{\lceil l / 3\rceil}}{2 r(2 r-1)^{(l-1)}} \leqslant \frac{1}{(\sqrt[3]{2 r-1})^{l}} .
$$

Now, Lemma 2.4 implies that the set of words $w$ for which the middle third of $w$ survives cyclic reduction of $w$ is exponentially generic in $\mathbb{F}$.

Proposition 2.8. Let $w$ be a word in $\mathbb{F}$. The set of words that contain $w$ as a subword of their cyclic reduction is exponentially generic in $\mathbb{F}$.

Proof. The set of words containing $w$ as a subword of their cyclic reduction contains the intersection of the set of words containing $w$ as a subword of their middle third with the set of words whose middle third survives cyclic reduction. By Lemma 2.6 and Lemma 2.7, both of these sets are exponentially generic in $\mathbb{F}$, so their intersection is as well.

Definition 2.9. Let $B \subset \mathbb{F}$. A word $w \in \mathbb{F}$ is poison to property $\mathcal{P}$ in $B$ if no word of $B$ containing $w$ as a subword of its cyclic reduction enjoys $\mathcal{P}$.

A word $w$ is (exponentially) generically poison to $\mathcal{P}$ in $B$ if there exists a (exponentially) generic subset $A \subset B$ such that $w$ is poison to $\mathcal{P}$ in $A$.

The following corollary is a consequence of Propositions 2.3 and 2.8.

Corollary 2.10. If there exists a word that is (exponentially) generically poison to $\mathcal{P}$ in $\mathbb{F}$ or $\mathbb{C}$ then $\mathcal{P}$ is (exponentially) rare in $\mathbb{F}$ or $\mathbb{C}$, respectively. 


\section{Whitehead graphs}

Definition 3.1. A multiword $\underline{w}=\left\{w_{1}, \ldots, w_{k}\right\}$ such that each $w_{i}$ is not a proper power, and such that $w_{i}$ is not conjugate to $w_{j}$ or $\bar{w}_{j}$ for all $i \neq j$, is called unramified.

A multiword is called cyclically reduced if all of its elements are cyclically reduced with respect to the fixed basis $\underline{x}$ of $\mathbb{F}$.

Let $\mathcal{T}$ be the Cayley graph of $\mathbb{F}$ with respect to $\underline{x}$, which is a $2|\underline{x}|$-valent tree. Let $\overline{\mathcal{T}}=\mathcal{T} \cup \partial \mathcal{T}$ denote the compactification of $\mathcal{T}$ by its Gromov boundary $\partial \mathcal{T}$.

Definition 3.2. If $\underline{w}$ is a cyclically reduced multiword, define $\mathbf{L}_{\underline{w}}$ to be the collection of distinct bi-infinite geodesics $\left[f \bar{w}^{\infty}, f w^{\infty}\right] \subset \overline{\mathcal{T}}$ where $w \in \underline{w}$ and $f \in \overline{\mathbb{F}}$.

DeFinition 3.3. Let $\underline{w}$ be a cyclically reduced multiword. Let $\mathcal{X}$ be a connected subset of $\mathcal{T}$. Let $\overline{\mathcal{X}}$ be its closure in $\overline{\mathcal{T}}$. The Whitehead graph of $\underline{w}$ over $\mathcal{X}$, denoted $\mathfrak{W}(\mathcal{X})$, is a graph whose vertices are in bijection with connected components of $\overline{\mathcal{T}} \backslash \overline{\mathcal{X}}$. Distinct vertices are joined by an edge for each $\mathcal{L} \in \mathbf{L}_{\underline{w}}$ with endpoints in the corresponding complementary components of $\overline{\mathcal{X}}$.

Remark. The Whitehead graph depends on $\underline{w}$ via $\mathbf{L}_{\underline{w}}$. We suppress $\underline{w}$ from the notation, as it will always be clear from context.

The following easy lemma clarifies the definition.

Lemma 3.4. Let $C_{1}$ and $C_{2}$ be components of $\mathcal{T} \backslash \mathcal{X}$. Then $C_{1}$ and $C_{2}$ are connected by an edge in $\mathfrak{W}(\mathcal{X})$ if and only if the label of the shortest path joining them is a subword of $w^{\infty}$ or $w^{-\infty}$ for some $w \in \underline{w}$.

REMARK. If $\underline{w}$ is unramified and cyclically reduced, and if $\mathcal{X}=*$ is a single vertex, then the vertices of $\mathfrak{W}(*)$ are in bijection with $\underline{x}^{ \pm}$, and there is one edge from vertex $\bar{x}$ to vertex $y$ for every occurrence of the subword $x y$ in $\underline{w}$, with words of $\underline{w}$ treated as cyclic words. This is the classical definition of the Whitehead graph.

Let $|w|$ denote the word length of $w$ with respect to the basis $\underline{x}$ of $\mathbb{F}$. Let $|[w]|$ denote the minimal word length of an element of the conjugacy class of $\underline{w}$.

Definition 3.5. An unramified, cyclically reduced multiword $\underline{w}=\left\{w_{1}, \ldots, w_{k}\right\}$ is Whitehead minimal if for every automorphism $\alpha \in \operatorname{Aut}(\mathbb{F})$ we have

$$
\sum_{i=1}^{k}\left|w_{i}\right| \leqslant \sum_{i=1}^{k}\left|\left[\alpha\left(w_{i}\right)\right]\right| .
$$

\section{Relative splittings}

Definition 4.1. A splitting of $\mathbb{F}$ relative to $\underline{w}$ is a splitting of $\mathbb{F}$ as a graph of groups such that each $w \in \underline{w}$ is elliptic.

The following lemma is essentially due to Whitehead [23]. See also $[\mathbf{1 6}, \mathbf{2 1}, \mathbf{2 2}]$.

LEmma 4.2. If $\mathfrak{W}(*)$ is connected and has no cut vertices then $\mathbb{F}$ does not split freely relative to $\underline{w}$.

The next lemma is a consequence of [4, Lemma 4.9]. 
Lemma 4.3. If $\mathbb{F}$ splits over $\langle v\rangle$ relative to $\underline{w}$ then $\mathfrak{W}\left(\left[\bar{v}^{\infty}, v^{\infty}\right]\right)$ has more than one connected component.

\section{Filling words and full words}

Kapovich and Lustig [11] define a non-trivial element $w \in \mathbb{F}$ to be filling if it has non-zero translation length for every very small isometric action of $\mathbb{F}$ on an $\mathbb{R}$-tree. (An action of $\mathbb{F}$ on an $\mathbb{R}$-tree is very small if tripod stabilizers are trivial and arc stabilizers are maximal cyclic.) Work of Guirardel [10] shows that $w$ is filling if and only if $F$ does not split freely or cyclically relative to $w$. Kapovich and Lustig ask for a combinatorial criterion that implies a word is filling. The first such criterion was given by Solie [20]. We will give another. Solie uses an exponentially generic set constructed by Kapovich, Schupp, and Shpilrain [12]. Essentially, the set consists of words that are balanced, in the sense that every element of $\underline{x}^{ \pm}$occurs roughly the same number of times in $w$ and every reduced two letter word in $\underline{x}^{ \pm}$occurs as a subword of $w$ roughly the same number of times (see [12, Proof of Theorem A]). Solie shows these words are filling. Our condition essentially says that a word is filling if it is sufficiently complicated, in the sense that every reduced three letter word in $\underline{x}^{ \pm}$occurs as a subword of $w$. Both Solie's condition and ours are satisfied on exponentially generic sets in $\mathbb{F}$; ours is somewhat simpler to check.

If $w$ and $v$ are words in $\underline{x}^{ \pm}$, we say $w$ cyclically contains $v$ if the free reduction of $v$ appears as a subword of the cyclic reduction of a power of $w$. We say a multiword $\underline{w}$ cyclically contains $v$ if one of the words of $\underline{w}$ cyclically contains $v$.

Definition 5.1. A multiword $\underline{w}$ is full if for every reduced word $v$ in $\left(\underline{x}^{ \pm}\right)^{3}$ either $v$ or $\bar{v}$ is cyclically contained in $\underline{w}$.

Lemma 5.2. The set of full words is exponentially generic in $\mathbb{F}$. The set of full, cyclically reduced words is exponentially generic in $\mathbb{C}$.

Proof. Let $B$ be either $\mathbb{F}$ or $\mathbb{C}$. Fix a reduced word $w$ containing every reduced word of $\left(\underline{x}^{ \pm}\right)^{3}$ as a subword. The set $A$ of words in $B$ whose cyclic reduction contains $w$ is exponentially generic in $B$, by Proposition 2.3 if $B=\mathbb{C}$ or Proposition 2.8 if $B=\mathbb{F}$.

The rest of this subsection is devoted to establishing that $\mathbb{F}$ cannot split freely or cyclically relative to a full word.

Lemma 5.3. The group $\mathbb{F}$ does not split freely relative to a full word.

Proof. Let $w$ be a full word. Its Whitehead graph contains the complete graph, so it is connected without cut vertices. By Lemma 4.2, $\mathbb{F}$ does not split freely relative to $w$.

Proposition 5.4. If $\gamma \subset \mathcal{T}$ is a line and $w$ is full, then $\mathfrak{W}(\gamma)$ is connected.

Proof. For $K \subset \gamma$ compact, let $C^{ \pm}$be the components of $\mathcal{T} \backslash K$ containing the rest of $\gamma$. Let $V_{K}$ be the vertex set of $\mathfrak{W}(K)$. Define $W(K)$ to be the full subgraph of $\mathfrak{W}(K)$ on vertices $V_{K} \backslash\left\{C^{ \pm}\right\}$.

Notice that for $K \subseteq K^{\prime}$ we have $W(K) \subseteq W\left(K^{\prime}\right)$, and moreover $\mathfrak{W}(\gamma)=\underline{\lim } W(K)$. The following claim thus suffices to establish the proposition.

Claim. $W(K)$ is connected, for any nonempty compact subsegment $K \subset \gamma$. 
Proof of Claim. We may suppose that $\gamma$ is parametrized to have unit speed, so that $\gamma$ sends integers to vertices of $\mathcal{T}$. For $z \in \mathbb{Z}$, let $s_{z} \in \underline{x}^{ \pm}$be the label of the edge $\left.\gamma\right|_{[z, z+1]}$.

The segment $K$ is equal to $\left.\gamma\right|_{[p, q]}$ for some integers $p, q$. Each vertex $C$ of $W(K)$ is a component of $\mathcal{T} \backslash K$, and there is a unique edge $e_{C}$ of $\mathcal{T}$ starting on $K$ and ending in $C$. We define $n_{C} \in \mathbb{Z}, s_{C} \in \underline{x}^{ \pm}$so that the initial point of $e_{C}$ is $\gamma\left(n_{C}\right)$, and the label of $e_{C}$ is $s_{C}$. The pair $\left(n_{C}, s_{C}\right)$ completely determines $C$, so we can also refer to the vertices of $W(K)$ by these pairs. Namely, $(n, s) \in \mathbb{Z} \times \underline{x}^{ \pm}$is a vertex of $W(K)$ if and only if

$$
p \leqslant n \leqslant q \text { and } s \notin\left\{\bar{s}_{n-1}, s_{n}\right\} .
$$

Two vertices $(n, s)$ and $(n, t)$ of $W(K)$ are connected by an edge if and only if one of $\bar{s} t$ or $\bar{t} s$ is a subword of $w^{\infty}$. Since $w$ is full, $(n, s)$ and $(n, t)$ are indeed connected by an edge. Two vertices $(n, s)$ and $(n+1, t)$ are connected if and only if $\bar{s} s_{n} t$ or its inverse occurs in some $w^{\infty}$. Again, since $w$ is full, all of these edges occur. It follows that $W(K)$ is connected.

COROLlary 5.5. The group $\mathbb{F}$ does not split freely or cyclically relative to a full word.

Proof. Let $w$ be a full word. Lemma 5.3 tells us there is no free splitting.

Suppose that $\mathbb{F}$ splits over $\langle v\rangle$ relative to $w$. Then by Lemma 4.3 the Whitehead graph $\mathfrak{W}\left(\left[\bar{v}^{\infty}, v^{\infty}\right]\right)$ has more than one connected component. But this contradicts Proposition 5.4.

In particular, fullness gives an easily verifiable, combinatorial condition implying that a word is filling, giving another answer to Kapovich and Lustig's question.

Corollary 5.6. A full word in $\mathbb{F}$ is filling.

\section{Virtual geometricity is rare}

Definition 6.1. For $x_{i} \in \underline{x}$ and $w \in \mathbb{F}$, an $x_{i}$-syllable is a maximal subword of $w$ equal to a power of $x_{i}$ or $\bar{x}_{i}$. A subword is a syllable if it is an $x_{i}$-syllable for some $i$.

Lemma 6.2 [2, Lemma p. 18]. Let $\underline{w}$ be unramified, cyclically reduced, Whitehead minimal and either geometric or non-orientably geometric. Suppose that $x_{1}$ and $\bar{x}_{1}$ do not form a separating pair of vertices in $\mathfrak{W}(*)$, and suppose that no $w \in \underline{w}$ begins and ends with distinct $x_{1}$-syllables. Up to absolute value, at most three different powers of $x_{1}$ appear as syllables of elements of $\underline{w}$.

Proof. Using a theorem of Zieschang [2, Theorem p. 11] one can show that if there were four we would be able to find four non-intersecting parallelism classes of properly embedded arcs in a punctured torus or punctured Klein bottle. An Euler characteristic argument shows that there are at most three such classes.

REMARK. Berge's [2] results are stated for orientable handlebodies, but the proofs of [2, Lemma p. 18] and Zieschang's theorem [2, Theorem p. 11] do not require orientability.

Theorem 6.3. Let $B$ be either $\mathbb{F}$ or $\mathbb{C}$. Let $\operatorname{VG}(l)$ be the probability that a word chosen randomly with uniform probability from the set of words of $B$ of length at most $l$ is virtually geometric. There exist $a>0$ and $b \in \mathbb{R}$ such that $\operatorname{VG}(l) \leqslant \exp (b-a l)$ for all sufficiently large $l$. 
Proof. Let $v^{\prime}$ be a reduced word with first letter $x_{1}$ and last letter $x_{2}$ that contains every word in $\left(\underline{x}^{ \pm}\right)^{3}$. Let $v=x_{1}^{1} x_{2} x_{1}^{2} x_{2} x_{1}^{3} x_{2} x_{1}^{4} x_{2} v^{\prime}$.

Let $C$ be the subset of $B$ consisting of words that are not proper powers and whose cyclic reduction is Whitehead minimal; $C$ is exponentially generic in $B$ by Theorem 2.2.

Let $w^{\prime}$ be a word in $C$ whose cyclic reduction $w$ contains $v$ as a subword. Then $w$ is full, so, by Corollary 5.5, $\mathbb{F}$ does not admit free or cyclic splittings relative to $w$. In particular, the JSJ decomposition of $\mathbb{F}$ relative to $w$ is trivial, so $[\mathbf{3}]$ implies $w$ is virtually geometric if and only if it is either orientably or non-orientably geometric. However, $\mathfrak{W}(*)$ contains the complete subgraph on its vertices, so it has no separating pairs of vertices. By Lemma 6.2, w cannot be geometric or non-orientably geometric, since at least four distinct powers of $x_{1}$ appear as syllables of $w$. Therefore, $w$ and $w^{\prime}$ are not virtually geometric.

We have shown that $v$ is exponentially generically poison to virtual geometricity in $B$, so the theorem follows from Corollary 2.10.

Theorem 6.3 is stated for single words. For multiwords, there are different models of genericity, but the presence of a single non-virtually geometric word in a multiword implies that the multiword is non-virtually geometric. Thus, virtual geometricity will be rare for any model in which a random multiword contains a long random word. We state corollaries for two popular models.

Corollary 6.4 ('Few Relators Model' of [1]). Let $B$ be either $\mathbb{F}$ or $\mathbb{C}$. For any $k \geqslant 1$, let $\operatorname{VG}(l)$ be the probability that a multiword consisting of $k$ randomly chosen words in $B$ of length at most $l$, selected independently and uniformly, is virtually geometric. There exist $a>0$ and $b \in \mathbb{R}$ such that $\operatorname{VG}(l) \leqslant \exp (b-a l)$ for all sufficiently large $l$.

Corollary 6.5 ('Density Model' of [9]). Let $B$ be either $\mathbb{F}$ or $\mathbb{C}$. For any density $0 \leqslant d \leqslant 1$, let $\mathrm{VG}(l)$ be the probability that a multiword consisting of $(2 \cdot \operatorname{rank}(\mathbb{F})-1)^{d l}$ randomly chosen words in $B$ of length at most $l$, selected independently and uniformly, is virtually geometric. There exist $a>0$ and $b \in \mathbb{R}$ such that $\operatorname{VG}(l) \leqslant \exp (b-a l)$ for all sufficiently large $l$.

Finally, we extract from the proof of Theorem 6.3 a non-virtual geometricity criterion.

Corollary 6.6. Let $\underline{w}$ be an unramified, Whitehead minimal, cyclically reduced multiword. If $\underline{w}$ is full and at least four distinct powers (up to absolute value) of some basis element occur as syllables of elements of $\underline{w}$, then $\underline{w}$ is not virtually geometric.

\section{Experimental estimates}

We wrote some computer scripts [5] to determine if a given multiword is virtually geometric or not. The underlying theory was developed in $[\mathbf{3}, \mathbf{4}, \mathbf{1 5}]$. We used these computer scripts to run computer experiments testing random words for virtual geometricity. The results are presented in this section. Let us first give a brief account of how the scripts work.

Given a multiword $\underline{w}$ and a word $v$, there is a way to determine if $\mathbb{F}$ splits over $\langle v\rangle$ relative to $\underline{w}$ by considering connectivity of certain finite generalized Whitehead graphs. (We improve Lemma 4.3 to only consider a bounded subsegment of $\left[v^{-\infty}, v^{\infty}\right]$.)

More specifically, we implement the algorithm of [4, Theorem 4.17] to search for splitting words. There are two ideas behind this search algorithm. First, there is a bound, depending on $\underline{w}$, of the maximal length of a cyclically reduced word $v$ such that the splitting of $\mathbb{F}$ over $\langle v\rangle$ relative to $\underline{w}$ is universal, that is, such that every other splitting word is elliptic with respect to this splitting. 

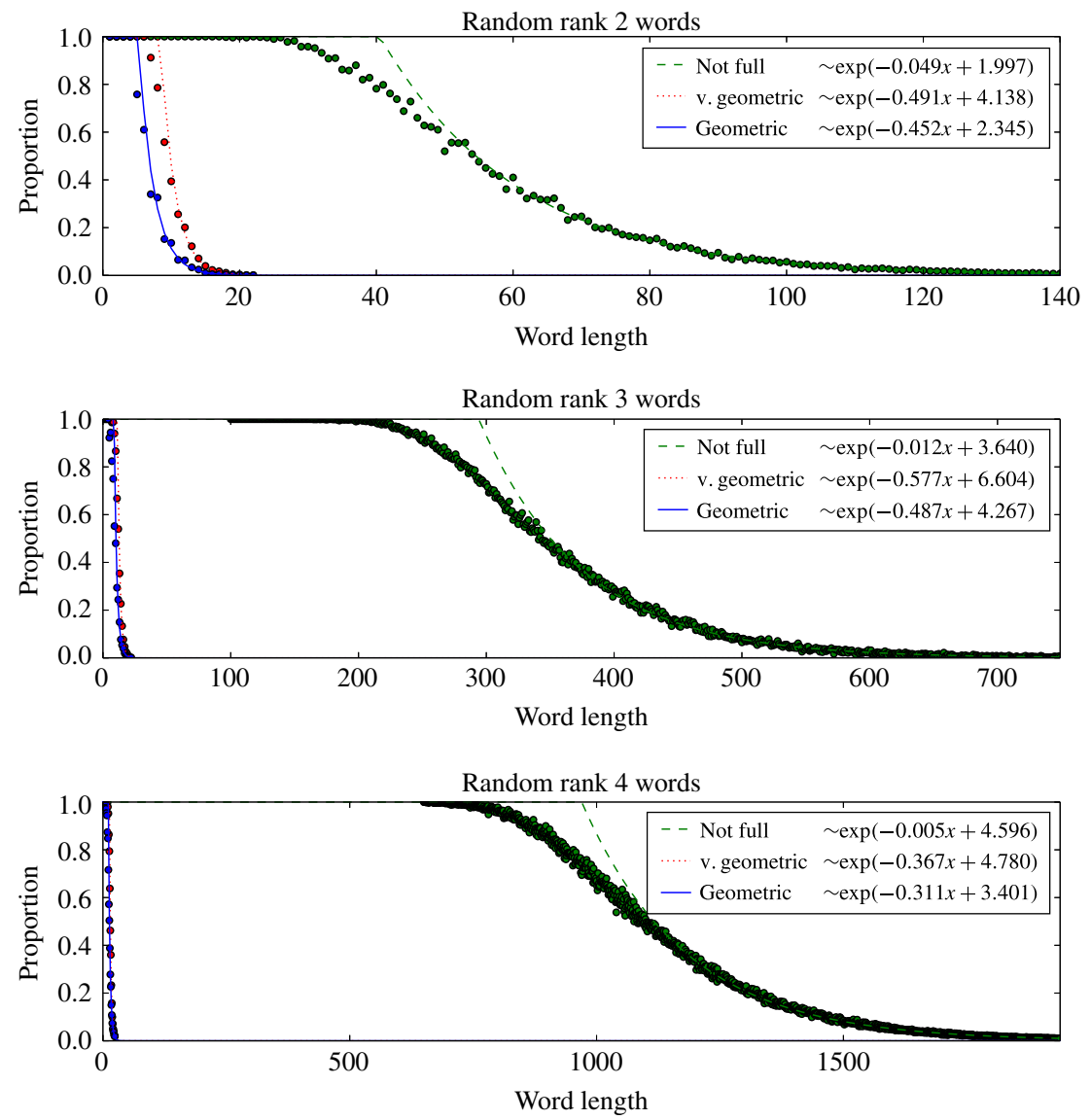

Figure 1. Geometricity, virtual geometricity, and full words.

Second, for a cyclically reduced splitting word, the generalized Whitehead graph over every prefix $p$ has a cut pair of a particular type, and this cut pair gives directions for finding the next letter of the splitting word. Thus, we can search inductively starting with short words $v$ and checking their generalized Whitehead graphs for these special cut pairs. If we find one, take the extensions $p x$ for $x \in \underline{x}^{ \pm}$suggested by the cut pairs.

The worst case estimates for the length of such a search are horrendous, but on generic multiwords it can be done effectively in low rank, because we quickly see that most short words can not be a prefix of a splitting word.

If $\mathbb{F}$ splits over $\langle v\rangle$, we deduce whether a second word $v^{\prime}$ is hyperbolic or elliptic in the splitting over $\langle v\rangle$ by adding edges corresponding to $v^{\prime}$ to the Whitehead graph over $v$ and checking if the number of connected components stays the same or decreases. We do this for all pairs of words found by the search algorithm to find the universal splitting words.

Given the list of conjugacy classes of universal splitting words, we compute the JSJ decomposition of $\mathbb{F}$ relative to $\underline{w}$, again using combinatorics of generalized Whitehead graphs. The main result of [3] says that $\underline{w}$ is virtually geometric if and only if the induced multiword in each vertex of the relative JSJ decomposition is orientably or non-orientably geometric. The induced multiword in a vertex group consists of conjugates of elements of $\underline{w}$ contained in that vertex group plus the image of a generator of each incident edge group.

The induced multiwords for quadratically hanging vertices of the relative JSJ decomposition are always geometric, so virtual geometricity of $\underline{w}$ is reduced to checking geometricity of the 

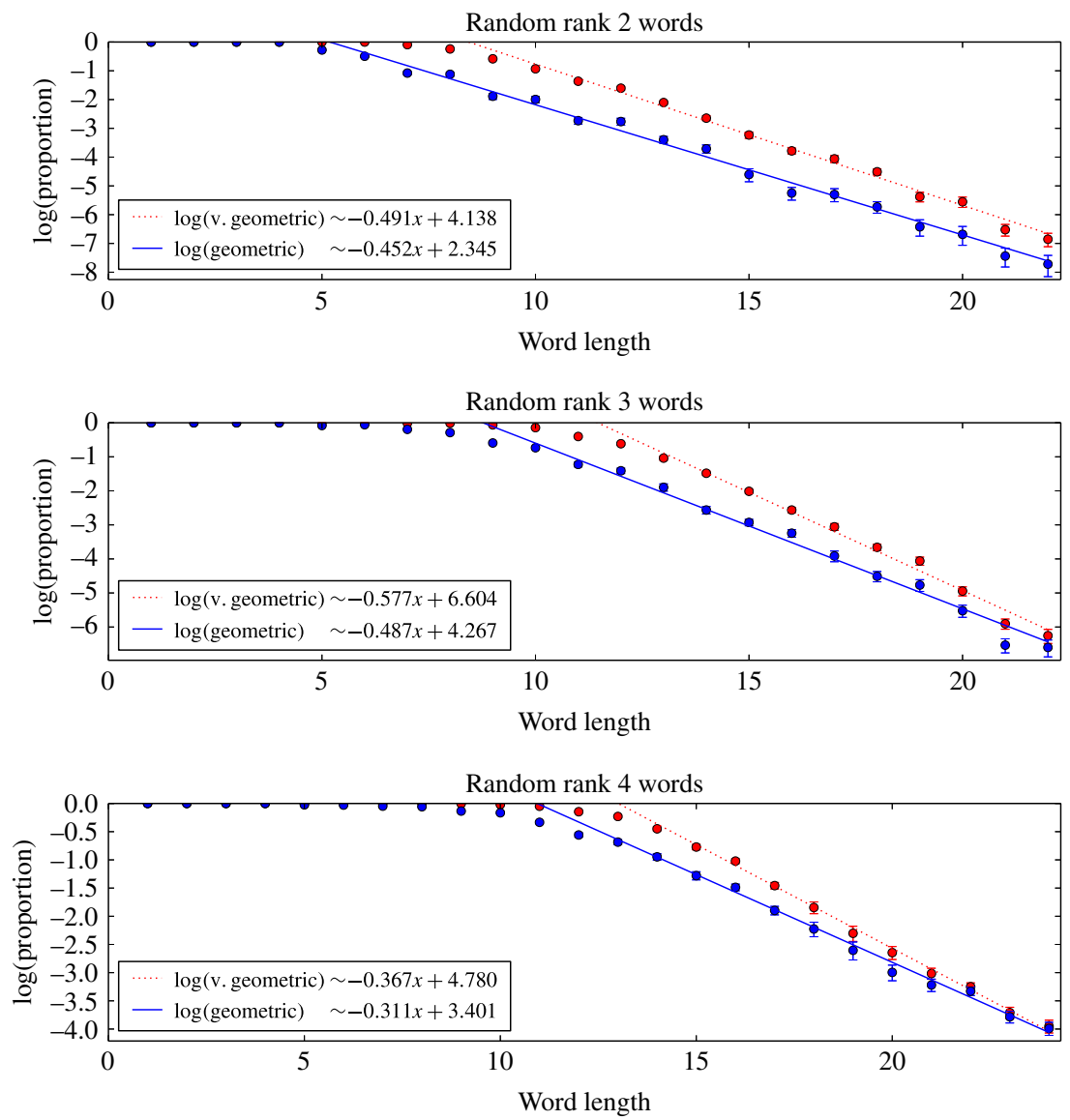

Figure 2. Geometricity and virtual geometricity.

induced multiword in each rigid vertex of the relative JSJ decomposition. For this we use Berge's program heegaard [2]. Berge shows that geometricity is equivalent to the existence of a planar embedding of the Whitehead graph of the multiword that satisfies an additional consistency requirement. The fact that we start with rigid vertices implies that such a Whitehead graph is 3-connected, so there is a unique planar embedding, if one exists. The heegaard program checks whether there exists a planar embedding, and, if so, finds it and checks the consistency conditions.

There is a slight complication that heegaard only checks the consistency conditions for orientable geometricity. To work around this issue, if heegaard says an induced multiword in a rigid vertex group is not geometric, we also check the lifts to all index 2 subgroups.

In the figures below we present findings of our computer experiments on the proportions of random words which are geometric, virtually geometric, and not full in ranks 2,3 , and 4 . (IPython [18] was used in the development of the computer scripts and in running the experiments. Scripts for testing virtual geometricity and reproducing our experiments can be found at https://bitbucket.org/christopher_cashen/virtuallygeometric.) Experiments on geometricity and virtual geometricity were performed before we found the proof of Theorem 6.3; the experiments on fullness were inspired by the proof.

We see in Figure 1 that while the proportion of not full words provides an exponentially decaying upper bound for the proportion of virtually geometric words, it is not very sharp. 
Fit curves are computed for each data series by taking the subseries that comes after the first word length for which the proportion of words falls below $50 \%$, taking logarithms, computing a best fit line by weighted least squares approximation, and then exponentiating.

Figure 2 plots logarithm (proportion \pm standard error) and omits the full words data. The number of trials increases with the word length so that the quantity

$$
\log \left(\frac{\text { proportion }+ \text { standard error }}{\text { proportion }- \text { standard error }}\right)
$$

stays small. Generating graphs with this amount of precision took about two months of continuous running time on a circa 2010 dual core desktop computer.

Acknowledgements. Thanks to Nathan Dunfield for pointing out Lemma 6.2, to John Mackay for explaining the note in Proposition 2.3, and to the referee for helpful comments and the reference to Solie's paper [20].

\section{References}

1. G. N. Arzhantseva and A. Yu. OL'Shanskir̆, 'Generality of the class of groups in which subgroups with a lesser number of generators are free', Mat. Zametki 59 (1996) no. 4, 489-496, 638, translated in Math. Notes 59 (1996), no. 3-4, 350-355, doi:10.1007/BF02308683; MR 1445193 (98k:20040).

2. J. Berge, Documentation for the program Heegaard, circa 1990, available at http://www.math.uic.edu/ $\sim \mathrm{t} 3 \mathrm{~m}$.

3. C. H. CAshen, Splitting line patterns in free groups, Preprint, 2010, arXiv:1009.2492v4.

4. C. H. Cashen and N. Macura, 'Line patterns in free groups', Geom. Topol. 15 (2011) no. 3, 1419-1475, doi:10.2140/gt.2011.15.1419; MR 2825316.

5. C. H. CASHen and J. F. MAnning, virtuallygeometric, 2014, computer program, available at https://bitbucket.org/christopher_cashen/virtuallygeometric.

6. C. Gordon and H. Wilton, On surface subgroups of doubles of free groups, Preprint, 2009, arXiv:0902.3693v1.

7. C. Gordon and H. Wilton, 'On surface subgroups of doubles of free groups', J. Lond. Math. Soc. (2) 82 (2010) no. 1, 17-31, doi:10.1112/jlms/jdq007; MR 2669638 (2011k:20085).

8. R. GrigorchuK and P. DE la Harpe, 'On problems related to growth, entropy, and spectrum in group theory', J. Dyn. Control Syst. 3 (1997) no. 1, 51-89, doi:10.1007/BF02471762; MR 1436550 (98d:20039).

9. M. Gromov, 'Asymptotic invariants of infinite groups', Geometric group theory, Vol. 2 (Sussex, 1991), London Mathematical Society Lecture Note Series 182 (Cambridge University Press, Cambridge, 1993) 1-295; MR 1253544 (95m:20041).

10. V. Guirardel, 'Approximations of stable actions on R-trees', Comment. Math. Helv. 73 (1998) no. 1, 89-121, doi:10.1007/s000140050047; MR 1610591 (99e:20037).

11. I. KAPOVich and M. Lustig, 'Intersection form, laminations and currents on free groups', Geom. Funct. Anal. 19 (2010) no. 5, 1426-1467, doi:10.1007/s00039-009-0041-3; MR 2585579 (2011g:20052).

12. I. Kapovich, P. Schupp and V. Shpilrain, 'Generic properties of Whitehead's algorithm and isomorphism rigidity of random one-relator groups', Pacific J. Math. 223 (2006) no. 1, 113-140; doi:10.2140/pjm.2006. 223.113; MR 2221020 (2007e:20068).

13. D. Lind and B. Marcus, An introduction to symbolic dynamics and coding (Cambridge University Press, Cambridge, 1995) doi:10.1017/CBO9780511626302; MR 1369092 (97a:58050).

14. J. M. MACKAy, 'Conformal dimension and random groups', Geom. Funct. Anal. 22 (2012) no. 1, 213-239, doi:10.1007/s00039-012-0153-z; MR 2899687.

15. J. F. Manning, 'Virtually geometric words and Whitehead's algorithm', Math. Res. Lett. 17 (2010) no. 5, 917-925, doi:10.4310/MRL.2010.v17.n5.a9; MR 2727618 (2012c:20114).

16. J.-P. Otal, 'Certaines relations d'equivalence sur l'ensemble des bouts d'un groupe libre', J. Lond. Math. Soc. s2-46 (1992) no. 1, 123-139, doi:10.1112/jlms/s2-46.1.123; MR 1180888 (93h:20041).

17. C. D. Papakyriakopoulos, 'On Dehn's lemma and the asphericity of knots', Ann. of Math. (2) 66 (1957) 1-26, MR 0090053 (19,761a).

18. F. PÉrez and B. E. Granger, 'IPython: a system for interactive scientific computing', Comput. Sci. Eng. 9 (2007) no. 3, 21-29, doi:10.1109/MCSE.2007.53.

19. P. Sсотт, 'Subgroups of surface groups are almost geometric', J. Lond. Math. Soc. (2) 17 (1978) no. 3, 555-565, MR 0494062 (58 \#12996).

20. B. B. Solie, 'Genericity of filling elements', Int. J. Algebra Comput. 22 (2012) no. 2, doi:10.1142/S02181 96711006741; MR 2903743. 
21. J. R. Stallings, 'Whitehead graphs on handlebodies', Geometric group theory down under (Canberra, 1996) (de Gruyter, Berlin, 1999) 317-330; MR 1714852 (2001i:57028).

22. R. Stong, 'Diskbusting elements of the free group', Math. Res. Lett. 4 (1997) no. 2, 201-210; MR 14530 54 (98h:20049).

23. J. H. C. Whitehead, 'On equivalent sets of elements in a free group', Ann. of Math. (2) 37 (1936) no. 4, 782-800, http://www.jstor.org/stable/1968618; MR 1503309.

Christopher H. Cashen

Fakultät für Mathematik

Universität Wien

Oskar-Morgenstern-Platz 1

1090 Vienna

Austria

christopher.cashen@univie.ac.at

\author{
Jason F. Manning \\ Department of Mathematics \\ 310 Malott Hall \\ Cornell University \\ Ithaca, NY 14853 \\ USA \\ jfmanning@math.cornell.edu
}

